\title{
负载型功能离子液体在有机合成中的应用研究进展
}

\author{
李胜男 $^{a}$ 赵雯辛 $^{a} \quad$ 刘玉静 $^{a}$ 刘中秋*, ${ }^{*}$ 应安国 $*, a, b$ \\ $\left({ }^{a}\right.$ 曲阜师范大学化学与化工学院 山东曲阜 273100) \\ ( ${ }^{b}$ 台州学院医药化工学院 浙江台州 318000)
}

\begin{abstract}
摘要 离子液体(ionic liquids, ILs)凭借其优良的物理化学特性以及对环境友好的特性, 得到广泛应用, 但 ILs 的高粘度 性导致反应后分离困难、可循环利用性低等问题. 而负载型功能离子液体(supported functional ionic liquids, SFILs)作为 离子液体和多种材料的结合体, 有着 ILs 和负载材料的双重优点, 可回收性能高、绿色经济高效, 在催化领域有着广泛 的应用. 主要对近几年几种不同类型的 SFILs 的制备、反应机理分析及其对反应的影响等研究成果进行了综述，包括负 载磁性纳米颗粒的 ILs、负载石墨烯、分子笁和有机一金属骨架等类型的 ILs,.
\end{abstract}

关键词＼cjkstart离子液体；载体；绿色化学; 催化; 环境友好

\section{Research Progress in the Application of Supported Functional Ionic Liquids in Organic Transformations}

\author{
Li, Shengnan $^{a} \quad$ Zhao, Wenxin $^{a} \quad$ Liu, Yujing $^{a} \quad$ Liu, Zhongqiu* ${ }^{* a} \quad$ Ying, Anguo* ${ }^{*, a}$ \\ ( ${ }^{a}$ Department of Chemistry and Chemical Engineering, Qufu Normal University, Qufu, Shandong 273100) \\ ( ${ }^{b}$ School of Pharmaceutical and Chemical Engineering, Taizhou University, Taizhou, Zhejiang 318000)
}

\begin{abstract}
Ionic liquids (ILs) have been widely used because of their excellent physical and chemical properties, and environment-friendly properties. However, the high viscosity of ILs causes difficulties in post-reaction separation and low recyclability. As the combination of ionic liquids with a variety of solid materials, supported functional ILs (SFILs) have the coupled advantages of ILs and loaded materials. It has a wide range of applications in the field of catalysis because of its high recovery performance, green economy and high efficiency. This paper mainly reviews the recent achievements over SFILs in respects of the preparation of ILs supported on various carriers like magnetic nanoparticles, graphene oxide, molecular sieves, organic-metal skeleton etc., the applications as the heterogeneous catalysts to organic transformations, and the understanding of the catalytic mechanism.

Keywords ionic liquids; carriers; green chemistry; catalysis; environmentally-friendly
\end{abstract}

近年来，人们对绿色环保、经济高效越来越重视， 这使研究出更多新型、绿色、高效的催化剂成为迫切需 要. 离子液体(ionic liquids, ILs)作为环境友好型之一的 催化剂和新型绿色环保溶剂已经广泛地运用于生物、化 学等领域. ILs 是由不对称的有机阳离子和有机或无机 阴离子组成的室温熔融盐, 具有独特的物理化学特性, 如可忽略的蒸汽压、高沸点、高化学稳定性和热稳定性、 不易燃烧性、良好的溶解性、可设计性等 ${ }^{[1]}$. 具有绿色 特性的 ILs 既可以替代传统易挥发性有机溶剂, 也可以 直接作为催化剂使用, 因此 ILs 也广泛地被应用在储能
材料 ${ }^{[2]}$ 、气体捕捉分 离 ${ }^{[3]}$ 、润滑剂添加剂 ${ }^{[4-5]}$ 、有机合 成 ${ }^{[6-7]}$ 等领域. 同时 ILs 作为溶剂有助于提高催化剂的活 性和对映选择性 ${ }^{[8]}$. 虽然 ILs 已被证实在化学反应过程 中效果显著，但其高粘度性导致只有少量的 ILs 参与反 应, 并且反应后与产物难分离, 使得 ILs 的可回收性下 降, 这些原因导致 ILs 的经济性和环境友好性下降. 如 何更好地解决这一难题, 也成为科研人员关注的地方.

随着负载型功能 ILs 概念的提出, 人们把焦点从单 独的 ILs 本身转移到负载型功能离子液体(supported functional ionic liquids, SFILs)上来. SFILs 是指通过共价

\footnotetext{
* Corresponding authors. E-mail: yinganguo@163.com; liuzhongqiuzs@126.com Received March 5, 2020; revised April 9, 2020; published online April 30, 2020. Project supported by the National Natural Science Foundation of China (Nos. 21978154, 21576176) and the National Students' Platform for Innovation and Entrepreneurship Training Programs (No. 201910446018).

国家自然科学基金(Nos. 21978154, 21576176)和国家级大学生创新创业训练计划(No. 201910446018)资助项目.
} 
键或非共价键固定在载体上的 ILs, 克服了 ILs 单独催化 反应时黏度大、成本高的缺点, 并保留 ILs 和载体特有 的优点 ${ }^{[9-12]}$. 由于 SFILs 是化学键合的方式存在, 避免 了在催化反应后 ILs 的流失, 提高了再生和循环使用性, SFILs 催化剂因其官能团丰富、良好的耐受性及绿色经 济高效性而受到广泛的关注 ${ }^{[13-14]}$. 另外, SFILs 在含有少 量 ILs 时便能达到单独使用 ILs 的催化效果, 节省了 ILs 的用量, 降低了制造成本, 更具经济环保性 ${ }^{[15-16]}$. SFILs 在催化化学反应时不仅能够提供良好的离子环境, 还可 以与反应底物形成较强的相互作用 ${ }^{[17]}$, 使催化效果更 优. 综合近几年不断涌现的科研成果发现, 国内外课题 组采用多种方法将 ILs 负载至不同载体材料上, 比如磁 性纳米颗粒 ${ }^{[29,42]}$ 、石墨烯 ${ }^{[48-51]}$ 、分子篮 ${ }^{[88-61]}$ 、有机一金属 骨架 ${ }^{[67-69]}$ 等, 制备了多种类型的 SFILs ${ }^{[18-20]}$, 大多数 SFILs 具有较高的比表面积、良好的催化活性及优异的 稳定性. 这些特性不仅有助于实现原料的高效转化, 还 有助于反应后原料与 SFILs 的分离, 这使得 SFILs 更多

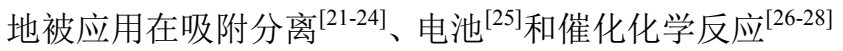
上来. SFILs 的应用使化学反应更加符合绿色化学理念.

本课题组一直致力于 SFILs 制备方法的优化与应 用 ${ }^{[29-32]}$, 且细致研究了其在反应中的催化性能及反应机 理. 本综述将参考本课题组与近几年国内外课题组在 SFILs 方面取得的部分成果, 综述 SFILs 制备方法、ILs 与催化剂的构效关系、催化剂的作用机理等, 希望为 SFILs 设计合成提供帮助与支持.

\section{2 负载型功能离子液体}

ILs 凭借其各种优异的物理化学特性, 在各大领域 得到广泛应用. 由于其不易分离以及高粘度的特性，使
SFILs 成为可能. SFILs 凭借自身拥有 ILs 和载体的双重 优点在催化领域得到广泛应用. SFILs 的应用大大提高 了反应的转化效果, 有着更优的收率, 更加环保高效.

\section{1 磁性氧化铁纳米颗粒负载型功能离子液体}

磁性纳米粒子(Magnetic Nanoparticles, MNPs) 一般 是由铁、钴、镍等金属氧化物组成的磁性内核及包裹在 磁性内核外的高分子聚合物、硅、羟基磷灰石壳层组成. MNPs 具有良好的稳定性、高比表面积、低毒性、合成 方法多样以及便于分离等独特的性能，是近年来发展迅 速且极具应用价值的新型材料，在现代科学的众多领域 如生物医药、磁流体、催化或者催化剂载体、核磁共振 成像、数据储存和环境保护等得到越来越广泛的应 用 ${ }^{[16,33-39]}$. 该类型的功能 ILs 主要和磁性氧化铁进行结 合制备催化剂, 使催化剂具有磁性, 便于从最终产物中 分离处理, 经过处理可以持续使用数次, 而且催化活性 没有显著降低.

本课题组 ${ }^{[29]}$ 设计了一种新型磁性纳米颗粒负载 ILs 的催化剂, 即 1,5,7-三氮杂二环 [4.4.0]癸-5-烯(Im-TBD( MNPs), 通过反应共沉淀的方法获得, 磁核为可回收 性提供了可能, 磁核外连接的硅壳防止催化剂的团聚现 象发生，同时外壳上的 $\mathrm{Si}-\mathrm{O}$ 键连接 ILs，增加催化剂总 体表面积, 提供更多的活性位点. 制备的催化剂可以在 无溶剂条件下催化 $N, N^{\prime}$-取代嫝的合成以及氮杂迈克尔 加成反应的发生. 该催化剂不仅适用于芳香胺与碳酸二 甲酯(DMC)或碳酸二乙酯(DEC)反应合成取代脲, 也适 用于环胺与各种复杂结构的 $\alpha, \beta$-不饱和羰基化合物的氮 加成反应(Scheme 1). 在相同反应条件下, Im-TBD@MNPs 催化反应可使产率达到 70\%, 比 TBD $@$ MNPs 和 TBD 催化反应达到的产率高出 $3 \% \sim 11 \%$. 由 Im-TBD@ MNPs 催化的 $N, N^{\prime}$-取代脲类化合物的合成以及环胺

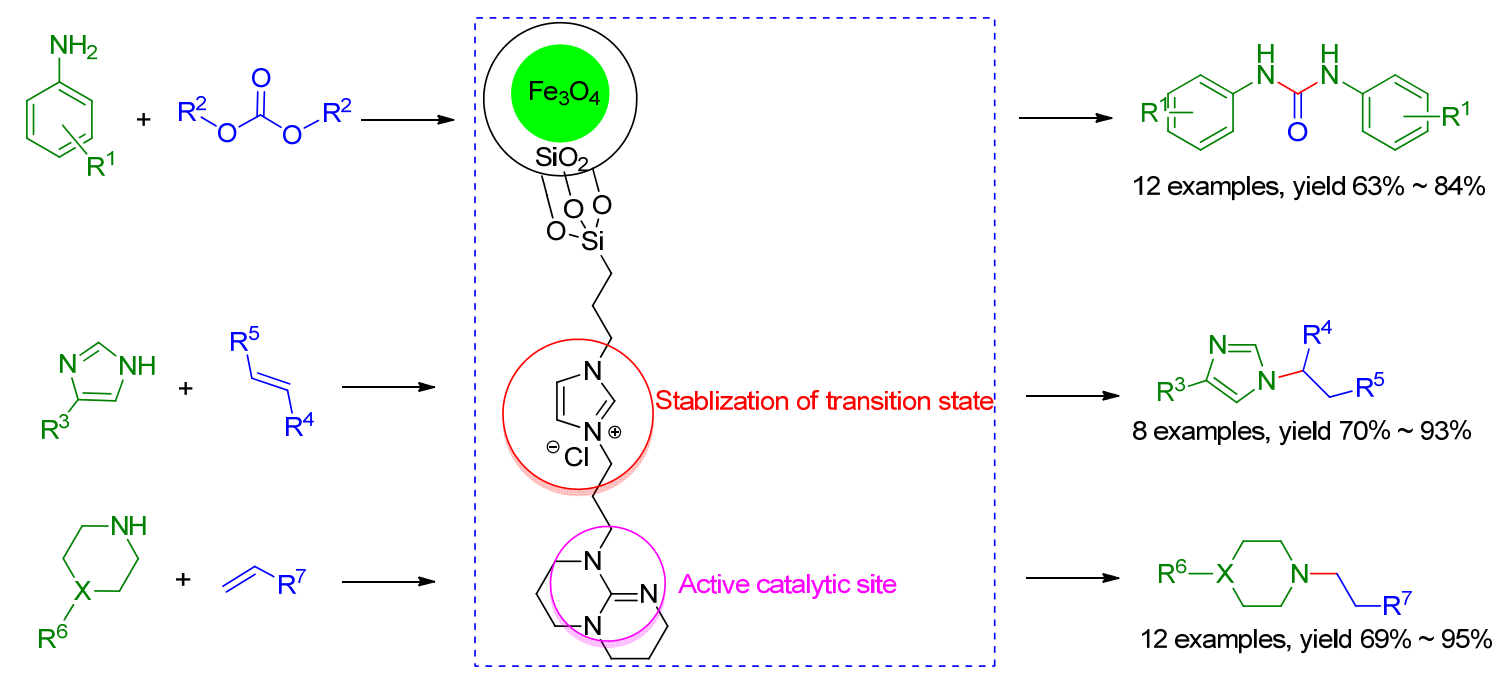

图式 $1 \mathrm{Im}-\mathrm{TBD} @ \mathrm{MNPs}$ 催化的各类反应

Scheme 1 Reactions catalyzed by Im-TBD@MNPs 
的氮杂-迈克尔加成反应的机理如 Eq. 1. 胍环上 $\mathrm{N}$ 原子 的孤立电子吸引胺中的氢原子, 从而增强 Michael 供体 的亲核性, 使活化的 $\mathrm{N}$ 亲核试剂与 $\mathrm{DMC}$ 或 $\alpha, \beta$-不饱和 羰基化合物顺利反应形成最终产品. 此外, “离子环境” 也可以加速反应的进行. 各种实验结果都很好地证明了 离子标记的 Im-TBD@MNPs 具有更好的催化性能, 这 促使我们开发更多具有离子标记类型的催化剂. 重要的 是，该催化剂可以通过外部磁铁吸附回收，重复使用 6 次没有显著的催化活性降低, 使有机反应更加绿色高 效.

Estakhri 等 ${ }^{[40]}$ 介绍了氯铝酸盐 ILs 修饰二氧化硅包 覆磁性纳米颗粒([ $\left.\mathrm{SiPrPy} \mathrm{AlCl}_{4} @ \mathrm{MNPs}\right)$ 的合成应用. 采 用溶胶一凝胶法制备 MNPs, 之后相继合成催化剂 [SiPrPy]- $\mathrm{AlCl}_{4} @ \mathrm{MNPs}$, 并在在无溶剂条件下高效催化 芳香醛、1,3-二酮和曲酸的一锅三组分合成各种二氢吡
喃并 $[3,2-b]$ 色二酮.

通过多种醛(取代苯甲醛、多环和杂芳烃) 1、曲酸 3 和 2 的一锅三组分反应, 合成了二氢吡喃并 $[3,2-b]$ 色二 酮 4、5 (Eq. 2). 在催化剂催化下，以良好的收率得到产 物 4、5, 产率范围在 $85 \% \sim 95 \%$.

由上述反应得到启发，该催化体系应用在从二醛选 择性合成单一和双-二氢吡喃并 $[3,2-b]$ 色二酮方面(Eq. 3) 也表现出优异的活性. 在催化剂的作用下，通过改变 $1 \mathrm{n}: 1 \mathrm{p}: 2 \mathrm{a}: 2 \mathrm{~b}: 3$ 的比例关系, 可以选择性地得到高 产率的单双二氢吡喃并 $[3,2-b]$ 色二酮. 催化剂主要催化 了醛 1 的活化, 并且同时催化 $\mathbf{2}$ 的烯醇式互变异构的发 生，最后才能和曲酸进行一锅法反应. 此外，反应完成 后，催化剂可以简单地使用外部磁铁分离，用氯仿洗 涤、干燥, 并重复使用. 催化剂重复使用 8 次, 均可得到 最终产物，反应时间和收率没有明显变化(92\%～95\%).
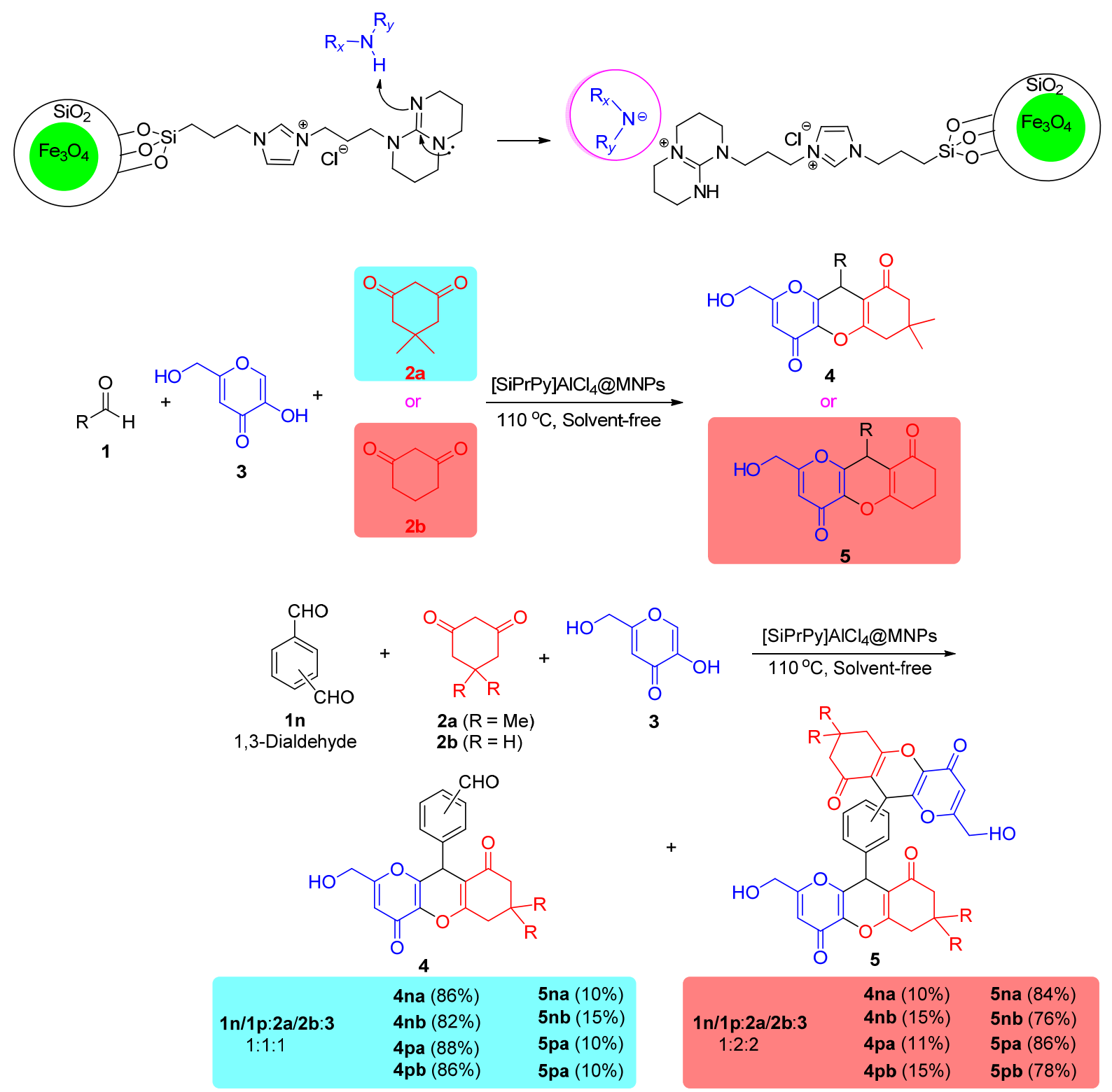
我们课题组 ${ }^{[30]}$ 设计合成了磁性纳米颗粒负载的咪 唑离子部分手性催化剂 6 , 成功地应用于对映体选择性 Michael 加成反应和不对称羟醛缩合反应中, 获得了优 良的产率 $(70 \% \sim 94 \%)$ 和令人满意的立体选择性 $(e e$ : $92 \% \sim 100 \%, d r: 91 ： 9 \sim 99 ： 1)$. 催化剂 6 的双功能性 在反应中不仅可以通过形成烯胺来增强醛或酮的亲核 活性, 而且可以提供酸性氢作为氢键供体来激活亲电试 剂, 以形成高立体选择性产物. 在以水为溶剂的异丁醛 和反式- $\beta$-硝基苯乙烯的不对称选择性反应中，6 催化反 应以较高的产率和良好的立体选择性得到产物, 但反应 时间过长，因此选择在 $20 \mathrm{~mol} \%$ 的催化剂 6 的存在下， 辅以 $20 \mathrm{~mol} \%$ 的 4-二甲氨基吡啶(DMAP)作为助催化剂, 反应时长缩短为 $4 \mathrm{~h}$, 获得的结果最佳. 而在相同的反 应条件下，同类型的催化剂 7 催化以水为溶剂的异丁醛 和反 式- $\beta$-硝基苯乙烯的不对称选择性反应中, 只得到 了 $18 \%$ 的产率和 $59 \%$ 的立体选择性. 催化剂 6 的手性诱 导以及离子部分的水合性质有效地促进了这种类型的 反应. 同时结合理论计算, 采用密度泛函的方法进行验 证, 结果和实验相对应. 在此基础上, 选择 6 作为环己 酮和芳香醛的不对称羟醛缩合反应的催化剂, 实验中的 醛都与环己酮顺利反应, 以良好的产率和高水平的选择 性得到了产物. 反应完成后, 催化剂可以使用外加磁力 分离和回收, 使用 5 次后, 没有明显的催化活性损失.
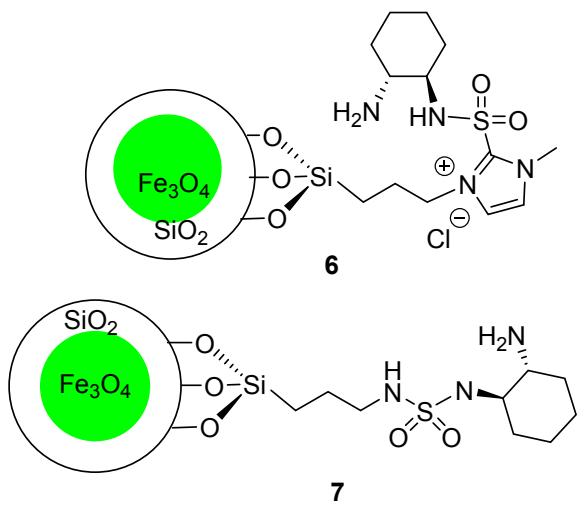

Teimuri-Mofrad 课题组 ${ }^{[41]}$ 合成了新型多相纳米催化 剂, 即二氧化硅包覆的 $\mathrm{Fe}_{3} \mathrm{O}_{4}$ 磁性纳米粒子负载的二茂 铁 ILs $\left(\mathrm{Fe}_{3} \mathrm{O}_{4} @ \mathrm{SiO}_{2}-\mathrm{IL}-\mathrm{Fc}\right)$, 同样采取了共沉淀和 Stober 方法制备了 $\mathrm{SiO}_{2}$ 包覆的 $\mathrm{Fe}_{3} \mathrm{O}_{4}$ 磁性纳米粒子. 在对比 ILs 和二茂铁 ILs 催化同一类反应时, 新合成的催化剂表 现最佳. 在超声波辐射下, 以乙醇和水(体积比为 $7: 3$ ) 为溶剂中获得的产率最佳, 通过各种醛 8 、丙二腈 9 和 曲酸或氯曲酸 10 在室温下的三组分反应(Eq. 4), 一锅法 合成了吡喃并 $[3,2-b]$ 吡喃衍生物. 在反应中, 首先酫和 丙二腈形成中间产物, 然后中间产物和酸进行 Michael 加成得到第二个中间产物, 最后经过环化得到最终产
物. 对比发现具有吸电子取代基的醛反应速度更快，而 具有给电子取代基的醛在反应中具有较低的反应活性, 所以反应时间变长. 利用 $\mathrm{Fe}_{3} \mathrm{O}_{4} @ \mathrm{SiO}_{2}-\mathrm{IL}-\mathrm{Fc}$ 作为催化剂 的催化反应, 可以缩短反应时长, 得到较高产率, 而且 催化剂易于制备. 更重要的是，反应完成后，催化剂用 乙醇处理并再次使用 6 次，催化活性仅损失 $5 \%$ ，更符合 绿色化学要求.

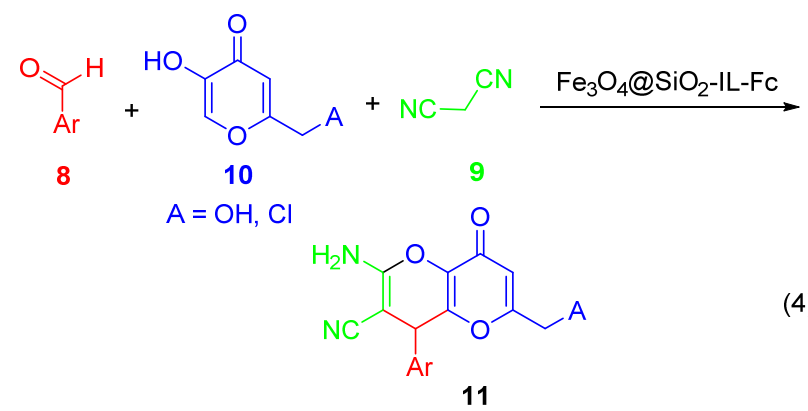

我们课题组 ${ }^{[31]}$ 合成了负载超顺磁纳米颗粒的咪唑 基 ILs 催化剂 Im@MNPs, 该催化剂可以高效催化芳香 醛与活性亚甲基化合物的 Knoevenagel 缩合反应. 特别 是在催化苯甲醛和丙二腈的的反应, $20 \mathrm{~min}$ 内便收获了 高达 $98 \%$ 的产率. 我们认为 $\operatorname{Im} @ M N P s$ 在催化体系中起 着双重作用(Scheme 2). 一方面, MNPs 表面裸露的羟基 与芳香醛形成氢键相互作用，使其更容易受到活性亚甲 基化合物的攻击. 另一方面，咪唑部分 $\mathrm{N}$ 原子的孤对电 子可以使活性亚甲基化合物去质子化，增强其亲核性. Im@MNPs 的双重催化作用在 Knoevenagel 缩合反应中 表现出了优异的催化性能. 此外, 该催化剂可以利用外 部磁铁轻易地从混合物中分离出来, 循环使用十次后催 化活性没有明显的下降.

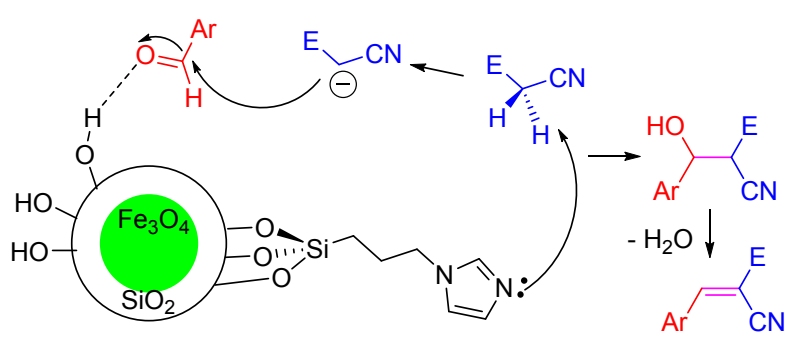

图式 2 由 Im@MNPs 催化的 Knoevenagel 缩合的合理机理 Scheme 2 A plausible mechanism for the Knoevenagel condensation catalyzed by Im@MNPs

Zohreh 课题组 ${ }^{[42]}$ 通过将铇酸盐阴离子固定在富氮 聚(ILs)/磁性纳米复合材料上, 合成了一种新型双功能 聚合物催化剂 MNP@PIL/W (12). 和多数催化剂的合成 方式相同，通过制备 $\mathrm{SiO}_{2}$ 包裹的磁性纳米粒子 MNPs, 然后将 MNPs 进行功能化处理, 最后通过蒸馏一沉淀聚 合法得到目标催化剂. 合成的聚合物催化剂上含有大量 
的铇酸盐和碱性氨基, 可以减少催化剂用量, 并且在一 锅合成反应中, 前期可以将醇选择性氧化为醛, 催化剂 表面存在的大量胺基使丙二腈去质子化, 同时催化剂表 面的离子基团可以促进缩合反应的进行, 所以可以很好 的促进醛和丙二腈之间的 Knoevenagel 缩合反应. 反应 在水介质中就可以观察到很好的产率, 主要得益于催化 剂表面的疏水亚甲基链起到了相转移的作用, 使得催化 剂在水介质中具有优异的氧化活性. 在以苯甲醇和丙二 腈为模型的反应中，使用 $5 \mathrm{~mol} \%$ 的 MNP@PIL/W 就可 以转化 $96 \%$ 以上的苯甲醇, 产生 $96 \%$ 的期望产品收率. 结果表明, MNP@PIL/W 对苯甲醇的氧化具有很高的选 择性, 在一锅法条件下能有效促进 Knoevenagel 缩合反 应(Scheme 3). 更重要的是, 反应后, 可以借助外部磁 力很容易地将催化剂分离出来, 并且 MNP@PIL/W 在 循环使用 5 次后催化活性没有明显下降.
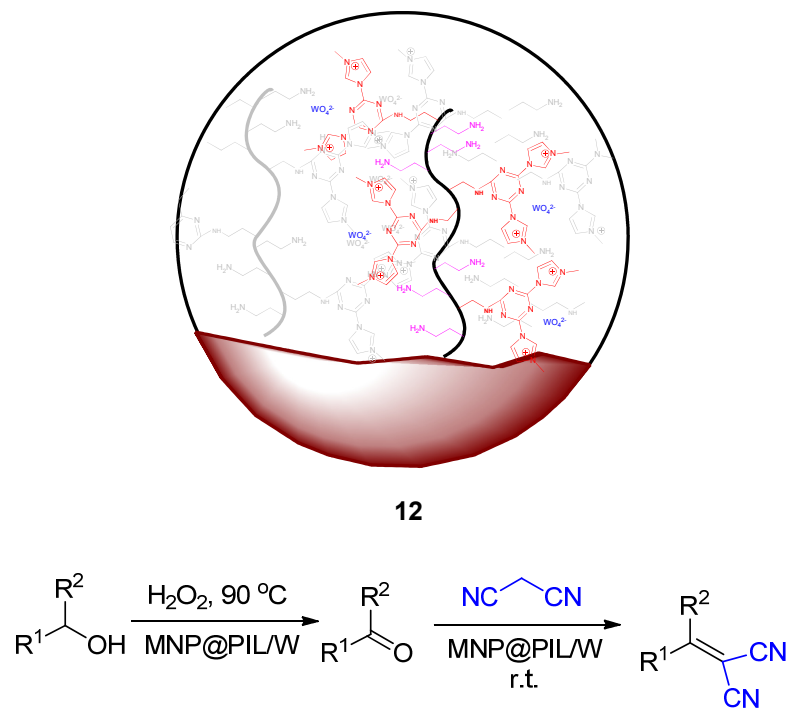

图式 $3 \mathrm{MnP} @ \mathrm{PIL} / \mathrm{W}$ 催化醇氧化/Knoevenagel 缩合的串联一 锅反应

Scheme 3 Tandem one-pot reaction of oxidation/Knoevenagel condensation of alcohols catalyzed by MNP@PIL/W

我们课题组 ${ }^{[32]}$ 制备了离子标记的磁性纳米颗粒 1,4二氮杂双环 [2.2.2]辛烷[IT-DABCO@MNPs, (13)], 一种 水溶性催化剂, 用于胺与 $\alpha, \beta$-不饱和酰胺的水相氮加成 反应. 该催化剂不仅有良好的催化活性, 而且还具有优 异的化学选择性. 在促进吗啉和 4-丙烯酰吗啉的共轭加 成反应中(Eq. 5), IT-DABCO@MNPs 表现出比有机碱更 好的催化活性. 在一系列脂肪胺和 $\alpha, \beta$-不饱和酰胺类的 反应中，离催化剂催化反应得到了 $75 \% \sim 89 \%$ 的产率， 但反应易受空间位阻的影响. 在反应中, 催化剂表面大 量的羟基和胺形成氢键, 有利于 $\mathrm{N}$ 阴离子的形成, 最后 作为亲核试剂和不饱和双键发生加成反应. 通过催化 4丙烯酰吗啉与 $N$-(2-差欮乙基)-哌嗪反应, 最终只检测到
$85 \%$ 收率的氮亲核攻击生成的产物(14), 该结果很好的 证明了 IT-DABCO@MNPs 在催化反应中的较高活性和 良好的化学选择性. 反应结束后, 用甲醇洗涤催化剂并 通过外部磁力回收，可重复使用 10 次，而催化活性没有 明显损失.
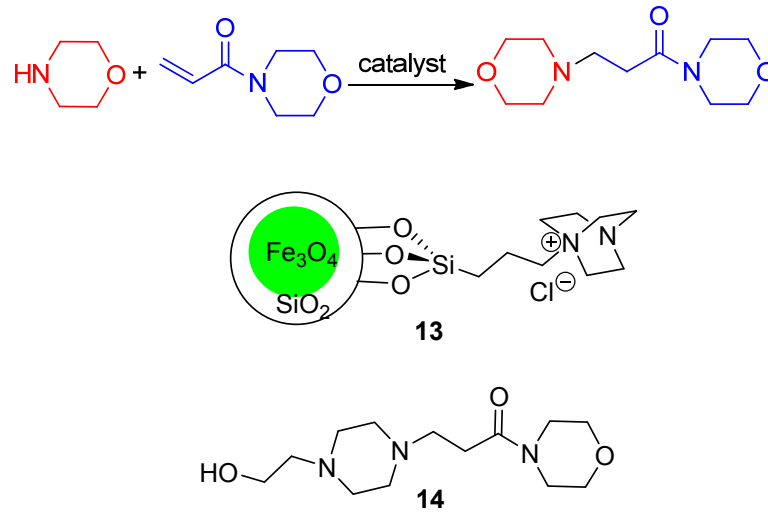

\section{2 氧化石墨烯(Graphene oxide, GO)负载型功能离 子液体}

近年来，石墨烯及其衍生物发展迅猛，应用范围广 泛. 作为石墨烯的衍生物, GO 具有高比表面积、高机械 强度和许多有利于 $\mathrm{GO}$ 进一步固定化的羟基官能 团 $^{[43-44]}$, 除此之外, $\mathrm{GO}$ 还具有耐酸和耐碱、高水热稳定 性、最小的传质限制等特性. $\mathrm{GO}$ 上的丰富的羟基还可以 和环氧化物形成氢键激活环氧化环, 促进开环 ${ }^{[45]}$, 因此 GO 作为一种理想的催化载体 ${ }^{[46-47]}$, 多应用于负载功能 化 ILs 形成高效催化剂, 此类型的催化剂兼具二者的优 点, 所以受到广泛应用.

Gaikwad 等 ${ }^{[8]}$ 设计合成了一种具有片状结构的新 型催化剂氧化石墨烯负载 Pd 金属和 1-甲基-3-(3-三甲氧 基硅丙基)咪唑氯 ILs(Pd@GOIL), 首先通过氧化法合成 $\mathrm{GO}$, 之后再将 ILs 和 $\mathrm{GO}$ 连接, 最后添加 $\mathrm{PdCl}_{2}$ 合成催 化剂Pd@GOIL. 该催化剂本身拥有有效的电子因素, 催化剂 Pd@GOIL 的扩展 X 射线吸收精细结构(EXAFS) 结果(图 1)表明, Pd 以 $\left[\mathrm{PdCl}_{4}\right]^{2-}$ 形式存在于 Pd@Go-IL 中, 正是由于 $P d$ 的这种形式存在，在促进羰基化反应中，可 以和更多不同给电子基团形成配合物，使得底物耐受范 围广, 可用于烷氧基、氨基、苯氧基的岸基化反应来合 成酯类和酰胺类化合物(Scheme 4). 与传统的羰基化反 应不同的是, 在温和的反应条件下, Pd@GOIL 催化过程 不需要添加任何助催化剂或者配体就能在短时间内得 到很好的效果 $(60 \% \sim 97 \%)$. 此外，该催化剂可循环回 收使用多达 8 次，并且没有明显的活性和选择性的损失.

李课题组 ${ }^{[49]}$ 合成了一种新型高效三相催化剂, 即负 载氧化石墨烯的吡啶 ILs (GO-Py-I). 首先采用改进的 


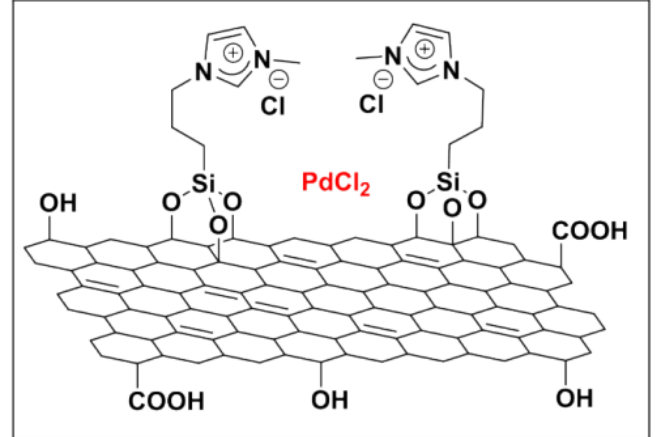

图 1 负载固定离子液体和 $\mathrm{PdCl}_{2}$ 离子的 $\mathrm{Pd} @ \mathrm{GOIL}$ 催化剂的 结构

Figure 1 Structure of Pd@GOIL catalyst bearing immobilized ionic liquid and $\mathrm{PdCl}_{2}$ ion

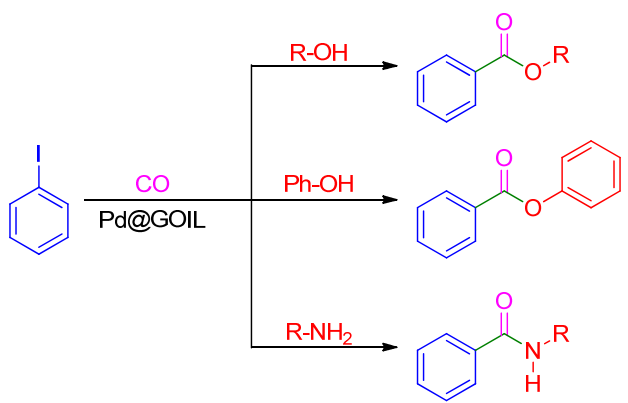

图式 4 Pd@GOIL 催化芳基碘的烷氧基、苯氧基和氨羰化反 应

Scheme 4 Pd@GOIL catalyzed alkoxy, phenoxy and aminocarbonylation of aryl iodide

Hummers 方法制备 GO, 并合成咪唑类和吡啶类 ILs, 之后将这两种 ILs 分别和 GO 连接制备了两种催化剂 GO-Im-X、GO-Py-X (X 代表卤素).由于这两种催化剂 所含有的阳离子电荷数的不同, GO-Py-X 的催化活性 要高于 GO-Im-X. ILs 的加入同时也大大提高了 GO 的 稳定性. 在同一类型催化剂中, GO-Py-I 的催化效果最 好，用于茮基卤化物与羊酸盐合成芐酯的亲核取代反 应(Eq. 6).

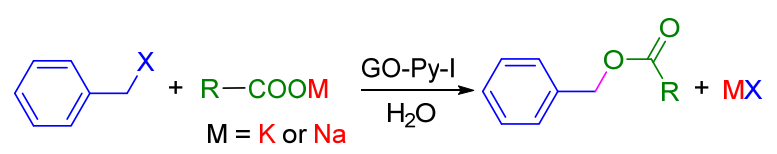

在液-固-液体系中, 羧酸根离子必须从水相迁移到 有机相，亲核取代反应才能顺利进行. 在水相中，GOPy-X 催化剂上的阴离子与羧酸盐中的阴离子发生交换, 形成活性离子对. 通过 PTCs 将羧酸根离子迁移到有机 相中. 然后, 卤化芐与羒酸盐在有机相中发生亲核取代 反应生成苄酯. 最后, GO-Py-X 催化剂进入水相, GO$\mathrm{Py}-\mathrm{X}$ 与羧酸盐再次进行离子交换. 在使用 GO-Py-I 作为 催化剂催化氯化茮和醋酸钾生成乙酸茮酯的反应中, 氯
化茮的转化率由空白实验下的 38\%提升至 99\%，但在不 同的底物中，长链羧酸钾更易使茮基卤化物获得高转化 率，而羧酸钾也比羧酸钠得到的转化率高. 但是总体来 看, GO-Py-I 在催化此类反应中表现出优异的催化活性. 这主要归功于 $\mathrm{GO}$ 的两亲性以及 ILs 对 GO 稳定性的影 响. 值得注意的是，该催化剂可以容易地回收和重复使 用至少五次，而没有明显的催化活性损失. GO-Py-I 的 合成大大解决了传统有机酯催化剂的污染大、分离难的 问题，让有机酯的合成变的更加环保、经济.

Sadjadi 课题组 ${ }^{[50]}$ 合成了由氧化石墨烯 $(\mathrm{GO})$ 、壳聚 糖(CS)、环糊精纳米 ILs(CDNS-IL) 以及 $\mathrm{Pd}$ 和 $\mathrm{Fe}_{3} \mathrm{O}_{4}$ 组 成磁性纳米催化剂 $\left(\mathrm{Fe}_{3} \mathrm{O}_{4} / \mathrm{Pd} @\right.$ Hybrid), 并将 $\mathrm{Fe}_{3} \mathrm{O}_{4} /$ $\mathrm{Pd} @$ Hybrid 用于 $\mathrm{Pd}$ 催化的硝基芳烃加氢反应和 $\mathrm{C}-\mathrm{C}$ 偶联反应. 在硝基苯的加氢反应中(Eq. 7), 能得到 100\% 的收率和转化率. 而且在更换不同硝基芳烃时，催化此 类反应同样可以得到高产率和转化率. 同时在 $\mathrm{Fe}_{3} \mathrm{O}_{4} /$ Pd@Hybrid 催化各种卤化物与末端炔烃、末端烯烃的 Sonogashira (Eq. 8)、Heck (Eq. 9)偶联反应中, 都能够在 短时间内得到较高产率. 该催化剂甚至还可以促进活性 较低的底物如氯苯和溴苯发生反应，分别达到 $70 \%$ 、 $60 \%$ 和 $90 \%$ 、80\%的产率. 该催化剂的优良性能主要源 于各组分的各自优良性能，比如在水中 GO 的低湿润 性、ILs、CS 增加了 Pd 的负载量和催化活性、CDN 包 裹疏水底物并将其转移到水介质中. 当然从经济环保性 来看，该催化剂最突出的特点是易于合成，而且可以在 反应后利用外部磁铁对其进行回收，但该催化剂在不失 去催化活性的情况下最多可以循环使用三次.
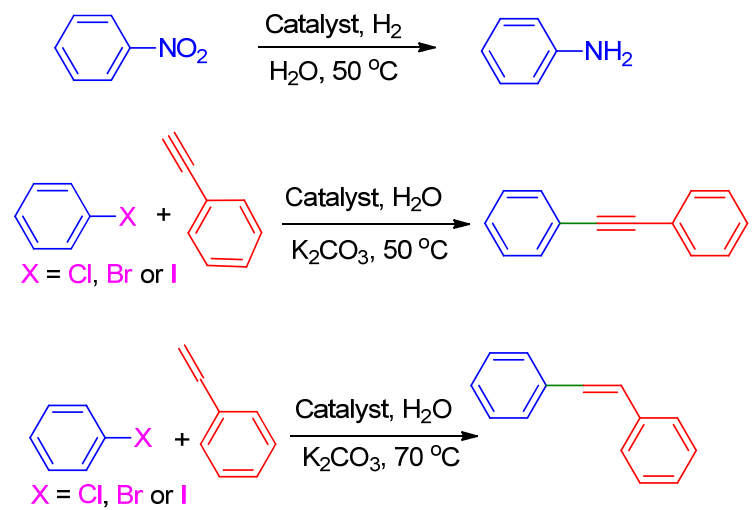

薛课题组 ${ }^{[51]}$ 开发了一种新方法来合成咪唑类 ILs 催 化剂(GO-[SmIm]X-HA- $n, n$ 代表酸度). 使用 Brønsted 酸 催化, ILs 可以通过环氧基的开环和羟基的硅烷化一步 负载到 $\mathrm{GO}$ 上, 由于较高酸度有利于环氧基团的开环, 从而大大提高了单位质量 GO 的 ILs 含量. 对比发现, 催 化剂 GO-[SmIm]X-HA-80 在催化同类型反应时，催化效 果最好. 将合成的催化剂应用于 $\mathrm{CO}_{2}$ 与环氧丙烷生成碳 
酸丙烯酯的环加成反应(Scheme 5), 环氧化物中的氧与 $\mathrm{GO}$ 上的差基形成氢键, 然后, 咪唑基 ILs 中的卤素阴离 子作为亲核试剂攻击环氧基上受阻碍小的碳原子使环 氧化物开环. 随后, 通过与带负电荷的氧反应, 将 $\mathrm{CO}_{2}$ 分子插入到该中间体中. 最后, 氢键断裂分子闭合成环 得到最终的环状碳酸酯. 而催化剂在循环使用五次后催 化活性略有下降. 此外该催化剂还可以催化 $\mathrm{CO}_{2}$ 和环氧 乙烷 $(\mathrm{EO})$ 、环氧氯丙烷 $(\mathrm{CPO})$ 、环氧苯乙烯 $(\mathrm{SO})$ 的环加 成反应，均获得了较高的转化率( $86 \%$ \% $98 \%$ ).

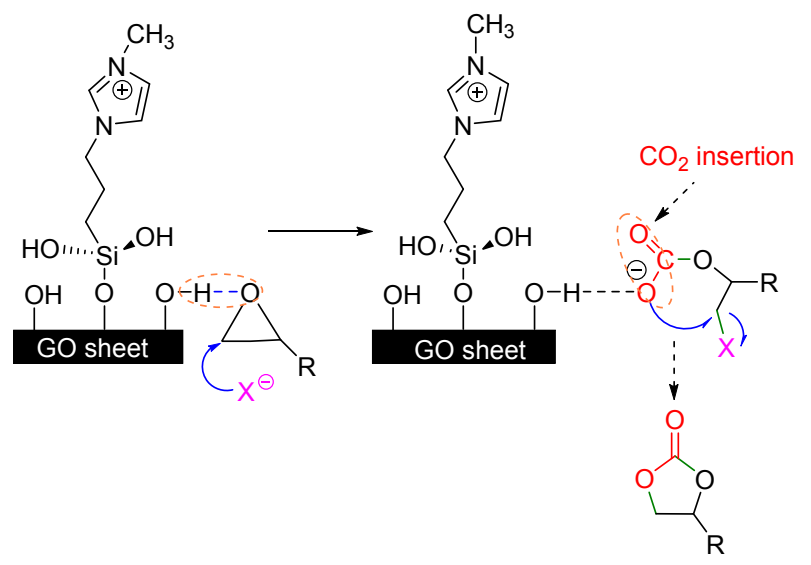

图式 5 GO-[SmIm]X-HA-80 催化合成环状碳酸酯 Scheme 5 Synthesis of cyclic carbonate catalyzed by GO[SmIm]X-HA-80

\section{3 分子篮负载型功能 ILs}

分子篮类的多孔材料 ${ }^{[52-54]}$ 在结构上有许多孔径均 匀的孔道和排列整齐的孔穴, 因此它的吸附能力高、选 择性强、耐高温. 较大的比表面积, 较强的酸中心, 足够 的表面硅醇基团 ${ }^{[55-57]}$, 晶孔内有强大的库仑场起极化作 用, 这些特性使得分子篮很多被用来作为催化剂载体, 其中分子篎负载的 ILs 由于具有良好的热稳定性和较大 的比表面积, 在用作多相催化剂催化反应时, 表现出了 高效的催化性能.

邱挺课题组 ${ }^{[58]}$ 合成了以 SBA-15 分子篎为载体的两 种负载型 ILs ([SBA-15-mim] $\mathrm{HSO}_{4}$, 图 2; [SBA-15-Ps$\mathrm{im}] \mathrm{HSO}_{4}$, 图 3), SBA-15 作为一种表面含着丰富硅氧基 才的介孔材料有着广泛的应用, 这里采用化学键合法将 两个离子液体固定在 SBA-15 上, 制备出一种新型催化 剂, 并将其作为催化乙酸仲丁酯(SBAC)和甲醇 $(\mathrm{MeOH})$ 酯交换合成 2-丁醇 $(\mathrm{SBOH})$ 反应的高效催化剂. 使用 [SBA-15-mim] $\mathrm{HSO}_{4}$ 和[SBA-15-Ps-im] $\mathrm{HSO}_{4}$ 催化该反应 时, SBAC 的转化率分别为 $64.9 \%$ 和 $89.1 \%$. [SBA-15-Ps$\mathrm{im}] \mathrm{HSO}_{4}$ 催化效率高的主要原因是烷基磺酸基团的存 在. 而使用 SBA-15 催化该反应时, SBAC 的转化率仅为 $1.4 \%$, 很明显新合成的催化剂具有更高的催化活性. 与
传统醇钠催化该反应相比, 虽然反应速率较低, 但最终 能收获与醇钠相当的收率，并且使用 [SBA-15-Ps$\mathrm{im}] \mathrm{HSO}_{4}$ 催化反应更加绿色环保. 此外, 该催化剂容易 回收，重复使用 5 次没有明显的活性损失，很好地证明 了该催化剂具有优异的重复使用性能和高催化活性，是 一种绿色环保型催化剂.

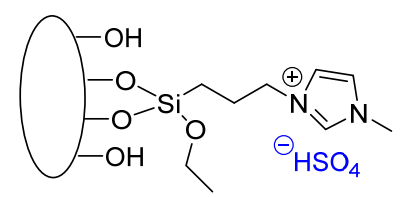

图 2 [SBA-15-mim] $\mathrm{HSO}_{4}$ 的结构

Figure 2 Structure of [SBA-15-mim] $\mathrm{HSO}_{4}$

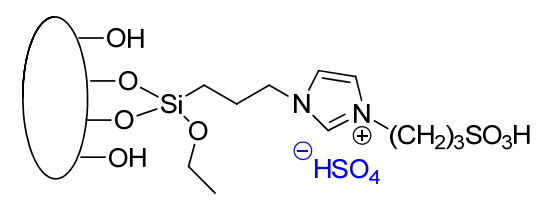

图 3 [SBA-15-Ps-im] $\mathrm{HSO}_{4}$ 的结构

Figure 3 Structure of [SBA-15-Ps-im] $\mathrm{HSO}_{4}$

郭课题组 ${ }^{[59]}$ 合成了 MCM-22 分子篮负载复合 ILs, 作为高效催化剂来催化二氧化碳和环氧丙烷的环加成 反应. 采用化学键合法, 将咪唑离子液体负载到分子篮 (MCM-22)上，制备了固定化离子液体催化剂，并将其 用于二氧化碳和环氧丙烷合成聚碳酸酯反应中. 在以功 能化的咪唑类 ILs 和 Lewis 酸 $\left(\mathrm{ZnBr}_{2}\right)$ 为原料合成一系列 MCM-22 分子笁负载的复合 ILs 中 $(\mathbf{1 5} \sim 18)$, MCM-22$[\mathrm{CeMIM}] \mathrm{Cl} /\left(\mathrm{ZnBr}_{2}\right)_{2}$ (18)催化效果最佳, 这是因为羧基 上的氢质子更容易在 MCM-22 的酸性中心内增强酸性, 为加速环氧化物的开环提供了有效的协同中心，同时酸 性的增加也会提高催化性能. MCM-22-[CeMIM]Cl/ $\left(\mathrm{ZnBr}_{2}\right)_{2}$ 催化反应机理(Scheme 6)主要是催化剂的羧基 端和 PO 形成氢键, 载体表面的负电荷作为亲核试剂攻 击环氧化物中位阻小的碳原子增加开环，有利于 $\mathrm{CO}_{2}$ 插 入，最后闭环形成 $\mathrm{PC}$, 释放催化剂，同时，固定在 MCM-22 上的 ILs 提高了催化剂的稳定性, 减少活性官 能团之间的相互作用, 避免了副产物的生成, 提高了选 择性. 当反应温度为 $120{ }^{\circ} \mathrm{C}$ 、压力为 $2.5 \mathrm{MPa}$ 、催化剂 用量为 $2.0 \%$ 时, 环氧丙烷的转化率为 $100 \%$, 选择性为 $97.4 \%$. 该催化剂在温和条件下可以循环使用 8 次.

丁辉课题组 ${ }^{[60]}$ 制备了 1-(3-磺酸盐)-丙基-3-烯丙基 咪唑硫酸氢(IL-1)、三氟甲基磺酸盐(IL-2)和磷铇酸二氢 (IL-3)三种 ILs，并且通过硫醇一烯反应分别将这三种 ILs 负载 SBA-15 分子篎到上, 形成稳定的 SILs-1、SILs-2、 SILs-3 催化剂. 

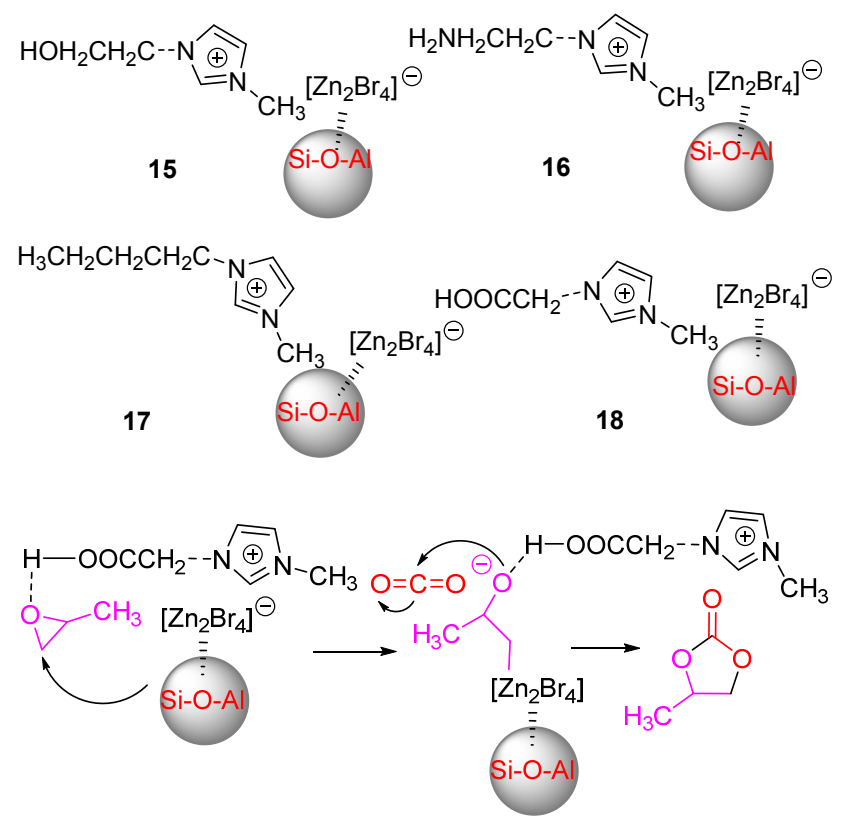

图式 $6 \mathrm{MCM}-22-[\mathrm{CeMIM}] \mathrm{Cl} /\left(\mathrm{ZnBr}_{2}\right)_{2}$ 催化反应机理研究 Scheme 6 Proposed reaction mechanism for MCM-22$[\mathrm{CeMIM}] \mathrm{Cl} /\left(\mathrm{ZnBr}_{2}\right)_{2}$ catalyzed reaction

三种 ILs 在催化棕榈酸酯化反应的酯化率均可达到 $90 \%$ 以上, ILs-2 催化棕㭣酸的转化效果明显高于另外两 种, 但是就和 SBA-15 固定化程度来说, IL-3 是负载率较 高(41.2\%)的酯化催化剂, 另外 IL-3 的大分子尺寸导致 了明显的空间位阻, 使得 IL-3 被负载在 SBA-15 的表面, 可以提供更多的活性催化位点，而且在最佳条件下， SILs-3 的酯催化效果可以高达 $88.1 \%$ (Eq. 7). 在催化棕 榈酸的酯化反应后, SIL-3 可以通过离心操作分离出来, 用乙醇处理后, 可以重复使用至少 5 次而没有明显的催 化活性损失. 应用在酯化预处理工艺中可以大大提高废 油质量, 更加经济环保.

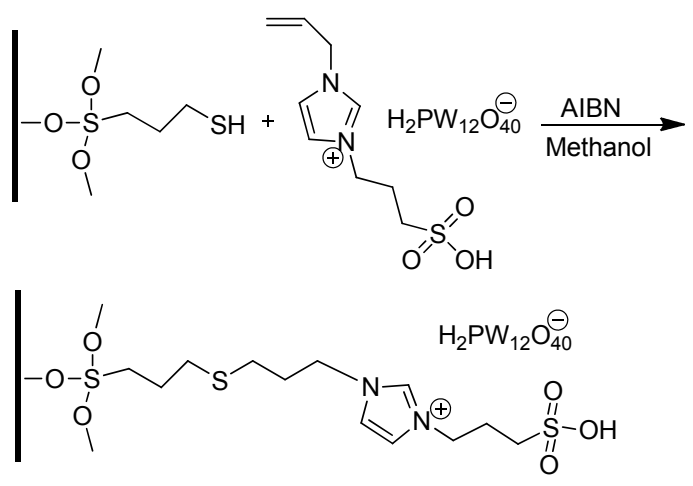

Hierro 课题组 ${ }^{[61]}$ 设计合成具有氢氧化胆碱 ILs 功能 的杂化介孔 Chol-SBA-15 (19), 并提出了一种新的高效 的硅胶表面连接方法, 采用共缩合法制备杂化介孔二氧 化硅微球(Chol-MSMs), 然后两步法合成了氢氧化胆碱 离子液体和 SBA-15 混合的材料(Chol-SBA-15), 以及以
氢氧化胆碱离子液体和六甲基二硅氮烷为封端剂的 SBA-15 杂化材料(Chol-HMDS-SBA-15), 并将所得的杂 化材料作为催化剂用于苯甲醛与丙二腈和氰乙酸乙酯 的 Knoevenagel 缩合反应(Eq. 8)中. 在室温和乙醇溶剂 中, 短时间内反应的转化率可以达到 $100 \% 、 89 \%$. CholHMDS-SBA-15 相对于 Chol-SBA-15 转化率更高, 主要 由于氢氧化物和 SBA-15 的相互作用, 降低表面剩余的 硅醇基数量. 但 Chol-SBA-15 主烷基链上羟基的存在同 样可以保证氢键的形成，释放的水增强了阴离子亚甲基 化合物的亲核加成. 另外, ILs 的存在也避免了疏水表面 对阴离子稳定性的影响. 虽然 Chol-MSMs 也有较好的 催化效果，但是回收性能远不如 Chol-SBA-15, 该催化 剂易于分离和回收，可重复使用 6 次也没有明显的活性 损失.
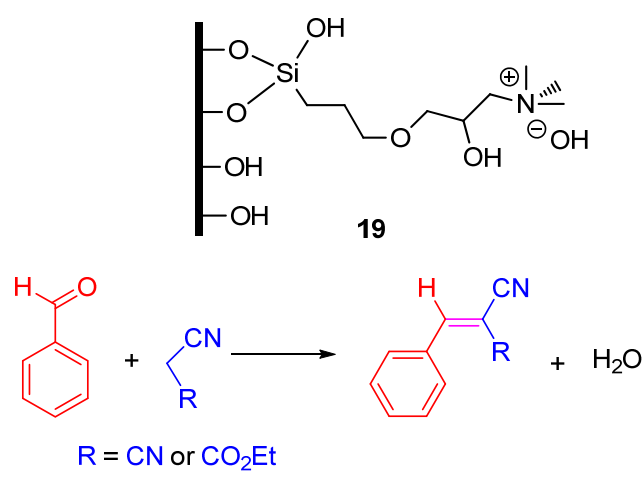

\section{4 金属-有机骨架(MOFs)负载型功能离子液体}

金属-有机骨架(MOFs) 是由含氧、氮等的多齿有机 配体(大多是芳香多酸和多碱)与过渡金属离子自组装而 成的配位聚合物. 大多数 MOFs 是以含羒基有机阴离子 配体为主，或与含氮杂环有机中性配体共同使用. 这些 金属有机骨架中多数都具有高的孔隙率和好的化学稳 定性、可控的孔结构 ${ }^{[62-64]}$ 、大的比表面积, 所以有着广 泛的应用. 这种新的多孔材料的特征，例如高孔体积、 低骨架密度等，与其他吸收材料(如多孔有机聚合物、沸 石和活性碳)相比，大的表面积使其拥有有效气体储存 的独特潜力 ${ }^{[65-66]}$. 所以用 MOFs 负载 ILs 作为催化剂, 既可以储存气体，又可以促进反应进行，除此之外，该 类型催化剂也便于从反应混合物中分离出来.

曹课题组 ${ }^{[67]}$ 采用一锅合成法首次合成了含有咪唑 基团、Lewis 酸性 $\mathrm{Cr}^{3+}$ 中心和游离卤素的介孔阳离子 Cr-MOF (FJI-C10). FJI-C10 可以完成对 $\mathrm{CO}_{2}$ 的大量吸附, 而且在常压和无助催化剂的情况下, 还能协同催化 $\mathrm{CO}_{2}$ 生成环状碳酸盐, 而其他类型 MOF 材料催化性能几乎 可以忽略. 在最佳反应条件下, FJI-C10 催化环氧化物和 $\mathrm{CO}_{2}$ 的反应中, 均能得到良好产率, 最高产率可达到 93\%. 催化机理主要是环氧化物被 Lewis 酸性的 $\mathrm{Cr}^{3+}$ 中 
心吸附活化, 然后, 卤素离子作为亲核试剂攻击环氧化 物受阻较小的一侧, 导致环氧化物开环, 之后 $\mathrm{CO}_{2}$ 插入 生成碳酸酯, 最后通过分子内环化反应生成环状碳酸酯 (Scheme 7). FJI-C10 催化底物范围广, 主要归因于限制 在介孔中的 $\mathrm{Cr}^{3+}$ 中心和咪唑基团的结合发挥协同作用. 另外 FJI-C10 可以通过简单的离心回收, 并且循环使用 6 次均能保持稳定的催化活性.

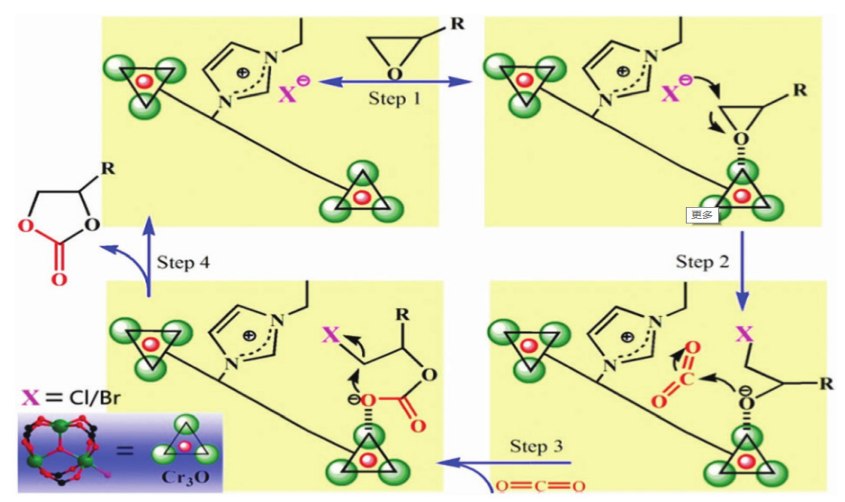

图式 7 FJI-C10 催化反应过程

Scheme 7 FJI-C10 catalytic reaction process

Shaabani 课题组 ${ }^{[68]}$ 设计合成了含有溴化胍负载金 属-有机骨架的高效催化剂[MIL101(Cr)-澳化胍], 在温 和、不使用任何助催化剂或有机溶剂的条件下, 成功催 化 $\mathrm{CO}_{2}$ 与环氧化物生成环碳酸酯的环加成反应, 并且获 得优异的催化效果. 不含溴化胍的 MOF 材料, 无法催 化环加成反应的发生, 而胍盐本身催化环加成反应效率 不高. 该催化剂巧妙地结合了三者的优点: $\mathrm{MOF}$ 对 $\mathrm{CO}_{2}$ 的高吸附性, MIL-101(Cr)中 Lewis 酸中心的助催化特性, 以及胍盐自身的催化性. 此外, 该催化剂可以重复使用 4 次, 没有明显的催化活性损失.

纪课题组 ${ }^{[69]}$ 采用 “瓶装法” 将 1-丁基-3-甲基咪唑 乙酸丁酯(BMIMOAc)ILs 固定在 MIL- $101 \mathrm{NH}_{2}$ 上, 制备 了一种新型的多相复合材料催化剂(IL(OAc $\left.{ }^{-}\right)$-MIL101$\mathrm{NH}_{2}$ ). 该催化剂表现出良好的催化活性, 在催化碳酸丙 烯酯与苯胺反应合成 3-芳基-2-噁唑烷酮 (20) 时, $\mathrm{IL}\left(\mathrm{OAc}^{-}\right)-\mathrm{MIL} 101-\mathrm{NH}_{2}$ 与碳酸丙烯酯的羰基氧相互作用 形成氢键, 然后活化后的苯胺和碳酸丙烯酯发生亲核反 应(21), 最终通过失去一个 $\mathrm{H}_{2} \mathrm{O}$ 分子和分子内环化得到 产物 20, 获得 92\%高产率. 它可以重复使用 6 次, 没有 明显的催化活性损失. 使用该催化剂催化碳酸丙烯酯与 芳香胺的反应, 均可以得到令人满意的收率 $(68 \%$ $92 \%$ ), 可以看出其耐受底物范围广泛. 总之, 该催化剂 的制备为合成 3-芳基-2-噁唑烷酮提供了一种简单、绿色 高效的方法.

\section{5 其他材料负载型功能离子液体}

除了上述类型的负载型 ILs, 另外还有很多优秀的

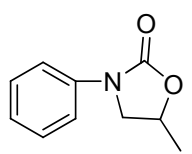

20

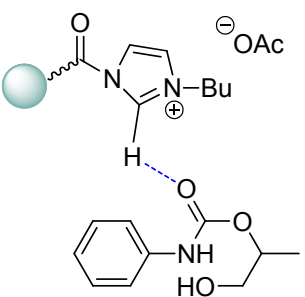

21
材料可以作为负载型多功能 ILs 的载体材料. 共价有机 骨架(COF)作为一种多孔晶体材料, 凭借低密度和高孔 隙率成为催化领域中的理想候选材料 ${ }^{[70-74]}$. 磷酸锆 $(\mathrm{ZrP}$, $\left.\mathrm{Zr}\left(\mathrm{HPO}_{4}\right)_{2} \bullet \mathrm{H}_{2} \mathrm{O}\right)$ 是一种多功能的层状化合物, 具有大的 离子交换容量和高度有序的层状结构、高密度的表面羟 基等特性 ${ }^{[75-76]}$, 而且易于合成, 其结晶度和大小可以很 好地控制 ${ }^{[77]}$, 所以多应用为聚合物填料、催化剂载体、 和润滑剂添加剂等 ${ }^{[78-81]}$. 壳聚糖(CS) 是一种天然的生物 聚合物，具有良好的生物相容性、生物降解性、无毒性 和良好的吸附性能特性. 此外, CS 可以很容易地进行化 学或物理改性, 可以作为优良的催化剂载体材料 ${ }^{[82-86]}$. 将这些材料和 ILs 结合起来作为催化剂, 能得到令人满 意的结果.

高课题组 ${ }^{[87]}$ 开发了一种在 COF 的通道壁上固定 ILs 的合成方法, 并合成了催化剂 $\left[\mathrm{Et}_{4} \mathrm{NBr}\right] x \%$-PyCOFs $(x \%$ 表示双醛混合物中存在的 DHPA 的摩尔分数), 当 $x=50$ 时, 效果最佳. 反应机理为: 在室温条件下, $\left[\mathrm{Et}_{4} \mathrm{NBr}\right]-$ $50 \%$-Py-COFs 作为双功能催化剂, 活化 $\mathrm{PhSiH}_{3}$ 与 $\mathrm{CO}_{2}$ 反应生成甲氧基硅烷，接着通过氢键活化胺(Scheme 8). 催化不同类型的胺与 $\mathrm{CO}_{2}$ 和 $\mathrm{PhSiH}_{3}$ 的无金属甲酰化反 应，以优异的产率 $(88 \% \sim 94 \%)$ 合成了不同类型的甲酰 胺. 此外该催化剂至少可以重复使用四次, 没有明显的 催化活性损失.

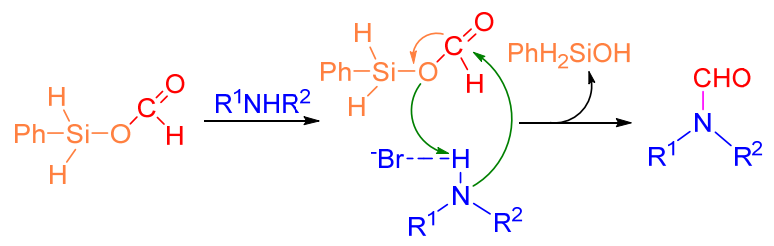

图式 $8\left[\mathrm{Et}_{4} \mathrm{NBr}\right] 50 \%-\mathrm{Py}-\mathrm{COFs}$ 催化剂催化甲酰化反应机理 Scheme 8 Mechanism of formylation catalyzed by $\left[\mathrm{Et}_{4} \mathrm{NBr}\right]-$ $50 \%$-Py-COFs catalyst

Sun 等 ${ }^{[88}$ 设计合成了一种高效、经济可循环催化剂 ZrP-SiIL, 利用 ZrP 纳米片表面的酸性羟基和功能化 ILs 上的硅烷基团之间的相互作用形成共价键来合成该催 化剂. 在无溶剂条件下, 将该催化剂用于催化苯甲酫与 氰乙酸乙酯的 Knoevenagel 缩合反应(Eq. 9), 获得优异 的收率 $(85.9 \% \sim 99.7 \%)$, 并且催化剂在反应后经过简单 
处理便可以继续使用, 没有明显的催化活性损失. 特别 是催化剂 $\mathrm{ZrP}(3 \mathrm{SiIL})$ ，它在 11 次循环后仍然保持了 $92 \%$ 的收率. 总之, 该类型的催化剂可以应用于 Knoevenagel 缩合反应, 对环境更加友好, 更能符合绿色化学理 念.

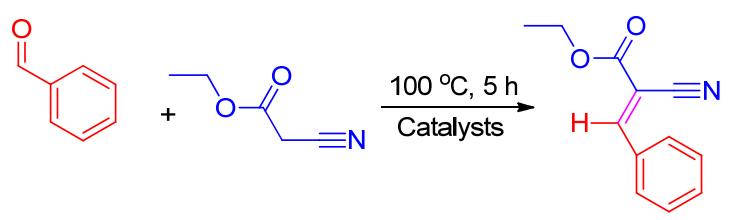

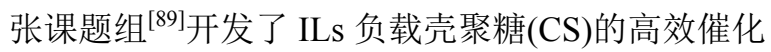
剂(CS-EMImX, $\mathrm{X}=\mathrm{Cl}, \mathrm{Br}$ ), 在不添加助催化剂和助溶 剂的情况下, 将其用于催化环氧化物和 $\mathrm{CO}_{2}$ 合成环状碳 酸酯的反应. 其中, 在最佳反应条件下, 合成的多相催 化剂 CS-EMImBr 具有较高的催化活性, 与均相 $\mathrm{EMImBr}$ 的产率相当. 部分归因于 CS 中含有羟基和叔 胺基团的 Lewis 碱对反应的协同催化作用.

催化机理如 Scheme 9. 首先, 环氧化物的氧与 CS 上的氢配位生成氢键, 卤化阴离子攻击环氧化物上位阻 小的碳发生开环, 同时, $\mathrm{CS}$ 上的叔氮原子与 $\mathrm{CO}_{2}$ 可逆配 位得到氨基甲酸盐, 开环后的环氧化物的氧负离子对氨 基甲酸盐进行亲核攻击产生烷基碳酸酯阴离子，最后， 通过分子内闭环形成环状碳酸酯, 催化剂释放再生. 另 外密度泛函理论计算结果也很好地证明了 CS 与 PO 环 上羟基之间的相互作用. 多相催化剂 CS-EMImBr 催化 底物范围广, 在催化 $\mathrm{CO}_{2}$ 与各种环氧化物的环加成反应 生成五元环碳酸酯的反应中, 获得较高的收率和转化率 和选择性. 反应后催化剂易于回收, 循环使用 5 次催化 活性和选择性没有明显下降.

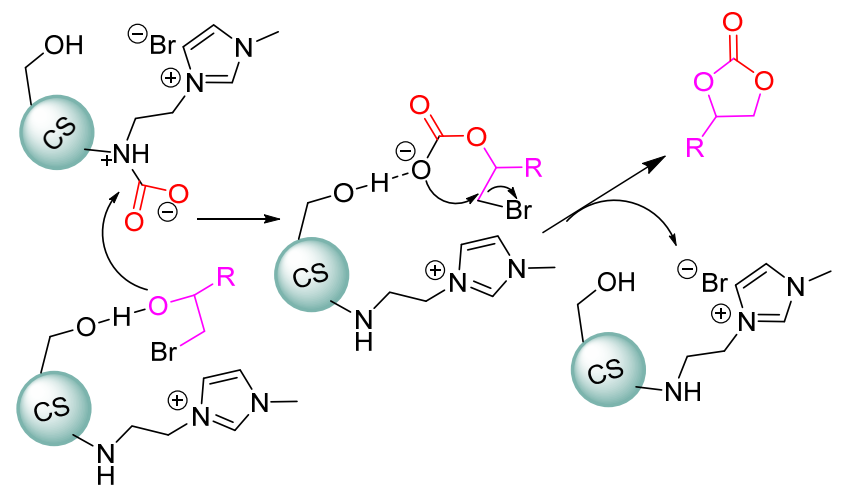

图式 9 CS-EMImBr 催化环加成反应机理

Scheme 9 Mechanism of cycloaddition reaction catalyzed by CS-EMImBr

Nassor 等 ${ }^{[90]}$ 制备了含 $\mathrm{NiMo} / \mathrm{C}$ 的磁性颗粒和 $\mathrm{Pd}$ 的 负载型 ILs 催化剂, 催化 1,5-环辛二烯(1,5-COD)和硝基 苯的加氢反应. 将得到的 SILP 催化剂(Pd- $1 \mathrm{IL} / \mathrm{NiMo} / \mathrm{C}$ 和 Pd-2.5IL/NiMo/C)用于 1,5-环辛二烯和硝基苯的加氢
反应. 对于 1,5-COD 的加氢反应, 催化剂 Pd-1IL/NiMo/ $\mathrm{C}$ 表现更佳, 比较两种催化剂的催化效果, 可以看出选 择性随 IL 层的增加而降低, IL 可能具有膜状效应, 有利 于有机相中间体环辛烯 $(\mathrm{COE})$ 的排出, 避免其与催化中 心接触. 而在对硝基苯的加氢反应中, $\mathrm{Pd}-2.5 \mathrm{IL} / \mathrm{NiMo} / \mathrm{C}$ 催化下的加氢反应可以得到更高的转化率, 对比发现随 着 IL 用量的增加, 转化率增加. 可能是 IL 和 NB 之间的 相互静电作用, 使得底物能够持久地靠近催化位置, 从 而有利于反应速度. 由此可以利用 IL 的影响来应用于 不同需求的反应, 所以可以利用外部磁铁进行分离, 解 决了催化剂分离难的问题.

谭课题组 ${ }^{[91]}$ 通过基于水相中蜡的 Pickering 乳液的 经典方法 (Scheme 10) 合成了 Janus 型催化剂[HO$\mathrm{SiO}_{2}$-IL-Ti(salen) $)_{x}, x$ 表示样品中的钛含量 $\left.\mathrm{mmol} / \mathrm{g}\right] \mathbf{2 2}$, 用于催化水相中不对称硫氧化反应. 当 $\mathrm{HO}-\mathrm{SiO}_{2}$-IL$\mathrm{Ti}$ (salen) $)_{0.07}$ 作为催化剂时，得到( $R$ )-甲基苯基亚砜的转 化率高达 $99 \%$, 化学选择性为 $97 \%, e e$ 为 $98 \%$. 而不含 IL 的催化剂在相同条件下只有 $80 \%$ 的中等转化率和较 低的选择性(94\%的化学选择性、ee 94\%). 除此之外, $\mathrm{HO}-\mathrm{SiO}_{2}-\mathrm{IL}-\mathrm{Ti}$ (salen) 0.07 还可以催化其他烷基芳基硫化 物在水中的不对称氧化反应，可以在 $1 \mathrm{~h}$ 内将底物定量 氧化为相应的亚砜，得到良好的转化率 $(86 \% \sim 99 \%)$ 和 对映体选择性 $(88 \% \sim 98 \%)$. 这些主要得益于两亲性 $\mathrm{HO}-\mathrm{SiO}_{2}$-IL-Ti(salen) $)_{x}$ 在乳化时产生高度分散的 Pickering 乳液, Pickering 乳液提供较大的油水界面 $(\mathrm{O} / \mathrm{W})$ 面积. 在乳化过程中, 使底物与周围环境隔离开，以创 造高浓度的催化环境; 同时, $\mathrm{HO}-\mathrm{SiO}_{2}$-IL-Ti(salen)将其 疏水的 Ti(salen)部分定向到油相(硫化物)中，确保反应 物和 $\mathrm{O} / \mathrm{W}$ 界面上的活性位点间有足够的接触面积，从 而促进反应的进行, 得到较高的转化率和化学对映体选 择性. 重要的是, 该催化剂可通过离心操作简易回收, 并且重复使用七次没有明显的活性、选择性损失. 该催 化剂的合成使得硫化物的氧化反应更加绿色高效.

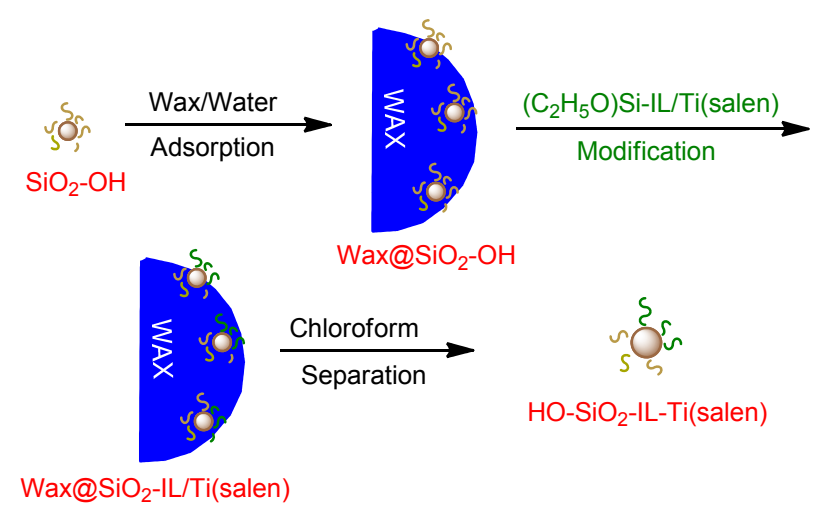

图式 $10 \mathrm{HO}-\mathrm{SiO}_{2}-\mathrm{IL}-\mathrm{Ti}(\text { salen) })_{x}$ 的合成 Scheme 10 Synthesis of $\mathrm{HO}_{-} \mathrm{SiO}_{2}$-IL-Ti(salen) 


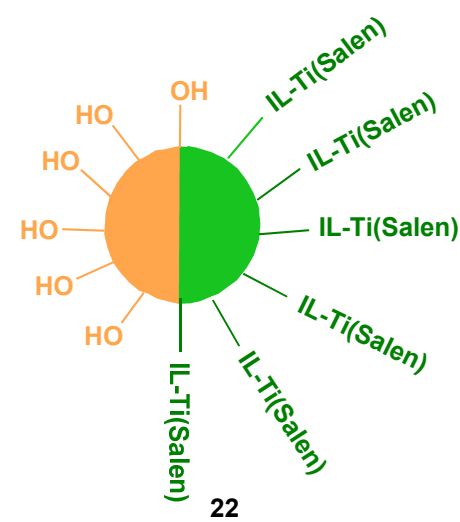

\section{3 结论}

综上所述, SFILs 大多数具有载体材料和 ILs 的双重 功能, 其作为催化剂在有机化学反应中已经得到了广泛 应用. ILs 和 MNPs 的结合解决了 ILs 单独作为催化剂粘 度高、分离难的问题, ILs 负载 GO 以增加催化剂中 ILs 的含量从而增加更多活性位点来提高催化活性, 而 ILs 负载分子篮等材料在催化反应时, 二者协同催化提高产 物收率. SFILs 可以简易回收、多次循环利用, 在使用少 量 ILs 合成的 SFILs 的情况下就可以达到更好的催化效 果, 甚至可以催化一些不能发生的反应, 使化学反应变 得更经济、高效. 解决了传统均相催化剂污染大、不可 循环的缺点, 也更加符合绿色化学的要求. 基于制备 SFILs 的载体材料种类繁多, 通过对比借鉴现有的载体 材料和不同 ILs 的催化特色, 为我们制备新型的催化剂 提供了更多的思考方向, 比如通过不同的载体材料和 ILs 的线性组合, 是否能够得到性能更加高效的催化剂.

但是大部分 SFILs 还停留在实验室阶段, 在现代工 艺中, 部分原料的制备技术并不成熟, 无法实现工业化 生产. 如何改善制备方法、节省制造成本、做好后续处 理、避免环境污染等依旧需要我们不断地探索. 只有解 决好这些难题, 才能使化学反应变的真正绿色起来, 使 得 SFILs 工业化成为可能, 使得我们未来的生活变得更 加美好.

\section{References}

[1] Wasserschied, P.; Keim, W. Angew. Chem., Int. Ed. 2000, 39, 3772.

[2] Watanabe, M.; Morgan, L. T.; Zhang, S.-G.; Ueno K.; Yasuda T.; Dokko, K. Chem. Rev. 2017, 117, 7190.

[3] Zeng, S.-J.; Zhang, X.-P.; Bai, L.; Zhang, X.-C.; Wang, H.; Wang, J.-J.; Bao, D.; Li, M.-D.; Liu, X.-Y.; Zhang, S.-J. Chem. Rev. 2017, 117,9625 .

[4] Zhou, Y.; Qu, J. ACS Appl. Mater. Inter. 2017, 9, 3029.

[5] Abhijeet, P.; Bapat.; Robert, E.; Bryan, T.; Seymour.; Zhao, B.; Cosimbescu, L. Eur. Polym. J. 2018, 108, 38.

[6] Karimi, B.; Vahdati, S.; Vali, H. RSC Adv. 2016, 6, 63717.

[7] Karimi, B.; Khorasani, M.; Naderi, Z.; Mirzaei, H. M.; Vali, H. Chem CatChem 2016, 8, 906.

[8] García, J, I.; Herrerías, C. I.; López-Sánchez, B.; Mayoral, J.A.;
Miñana, A. C. Tetrahedron: Asymmetry 2014, 25, 833.

[9] Giacalone, F.; Gruttadauria, M. ChemCatChem 2016, 8, 664.

[10] Mehnert, C. P. Chem. Eur. J. 2005, 11, 50.

[11] Van, D. C.; Wahlen, J.; Mertens, P.; Binnemans, K.; Vos, D. D. Dalton Trans. 2010, 39, 8377.

[12] Giacalone, F.; Gruttadauria, M. Chem CatChem 2016, 8, 664.

[13] Xu, J.; Xu, M.; Wu, J.; Wu, H.; Zhang, W.-H.; Li, Y.-X. RSC Adv. 2015, 5, 72361.

[14] Xu, B.-H.; Wang, J.-Q.; Sun, J.; Huang, Y.; Zhang, J.-P.; Zhang, X.-P.; Zhang, S.-J. Green Chem. 2015, 17, 108.

[15] Mehnert, C. P. Chem. Eur. J. 2005, 11, 50.

[16] Zheng, X.-X.; Luo, S.-Z.; Zhang, L.; Cheng, J.-P. Green Chem. 2009, 11,455

[17] Karimi, B.; Mansouriand, F.; Vali, H. Green Chem. 2014, 16, 2587.

[18] Yang, J.-B.; Zhou, L.-H.; Guo, X.-T.; Li, L.; Zhang, P.; Hong, R.-Y.; Qiu, T. Chem. Eng. J. 2015, 280, 147.

[19] Wang, B.; Zhang, J.; Zou, X.; Dong, H.; Yao, P. Chem. Eng. J. 2015, 260, 172 .

[20] Severa, G.; Bethune, K.; Rocheleau, R.; Higgins, S. Chem. Eng. J. 2015, 26, 249.

[21] Askalany, A. A.; Freni, A.; Santori, G. Desalination 2019, 452, 258.

[22] Jebur, M.; Sengupta, A.; Chiao, Y. H.; Kamaz, M.; Qian, X.-H.; Wickramasinghe, R. J. Membr. Sci. 2018, 556, 1.

[23] Zhu, J.-M.; He, B.-T.; Huang, J.-H.; Li, C.-C.; Ren, T. Microporous Mesoporous Mater. 2018, 260, 190.

[24] Uehara, Y.; Karami, D.; Mahinpey, N. Energy Fuels 2018, 32, 5345.

[25] Mendes, T. C.; Zhang, X-M.; Wu, Y-T.; Howlett, P. C.; Forsyth, M.; Macfarlan, D. R. ACS Sustainable Chem. Eng. 2019, 7, 3722.

[26] Mehnert, C. P.; Mozeleski, E. J.; Cook, R. A. Chem. Commun. 2002, 3010 .

[27] Lee, C.; Sandig, B.; Buchmeiser, M. R.; Haumann, M. Catal. Sci. Technol. 2018, 8, 2460.

[28] Oriol, M. F.; Chacón, G.; Bernardi, F.; Grehl, T.; Brüner, P.; Dupont, J. Catal. Sci. Technol. 2018, $8,3081$.

[29] Ying, A.-G.; Hou, H.-L.; Liu, S.; Chen, G.; Yang, J.-G.; Xu, S.-L. ACS Sustainable Chem. Eng. 2016, 4, 625.

[30] Ying, A.-G.; Liu, S.; Li, Z.-F.; Chen, G.; Yang, J.-G.; Yan, H.; Xu, S.-L. Adv. Synth. Catal. 2016, 358, 2116.

[31] Li, Z.-F.; Hu, H.-N.; Jin, Y.-X.; Li, R.-R.; Ying, A.-G.; Xu, S.-L. Curr. Org. Synth. 2015, 12, 467.

[32] Ying, A.-G.; Liu, S.; Ni, Y.-X.; Qiu, F.-L.; Xu, S.-L.; Tang, W.-Y. Catal. Sci. Technol. 2014, 4, 2115.

[33] Abolfazl, A.; Samiei, M.; Davaran, S. Nanoscale Res. Lett. 2012, 7, 144.

[34] Polshettiwar, V.; Luque, R.; Fihri, A.; Zhu, H.; Bouhrara, M.; Basset, J. M. Chem. Rev. 2011, 111, 3036.

[35] Gawande, M. B.; Branco, P. S.; Varma, R. S. Chem. Soc. Rev. 2013, 42, 3371.

[36] Baig, R. N.; Varma, R. S. Green Chem. 2013, 15, 398.

[37] Sadeghzadeh, S. M.; Daneshfar, F.; Malekzadeh, M. Chin. J. Chem. 2014, 32, 349.

[38] Lee, J.; Chung, J.; Byun, S. M.; Kim, B. M.; Lee, C. Tetrahedron 2013, 69, 5660 .

[39] Hu, H.; Liu, S.; Lin, W. RSC Adv. 2012, 2, 2576.

[40] Estakhri, E. Nasr-Esfahani, M.; Mohammadpoor-Baltork, I.; Tangestaninejad, S.; Moghadam, M.; Mirkhani, V. Appl. Organomet. Chem. 2017, 31, 3799.

[41] Teimuri-Mofrad, R.; Esmati, S.; Rabiei, M.; Gholamhosseini-Nazari, M. Heterocycl. Commun. 2017, 23, 439.

[42] Zohreh, N.; Tavakolizadeh, M.; Hosseini, S. H.; Pourjavadi, A.; Bennett, C. Polymer 2017, 112, 342.

[43] Eigler, S.; Hirsch, A. Angew. Chem., Int. Ed. 2014, 53, 7720.

[44] Gómez-Navarro, C.; Burghard, M.; Kern, K. Nano Lett. 2008, 8, 2045.

[45] Saptal, V. B.; Sasaki, T.; Harada, K.; Nishio-Hamane, D.; Bhanage, B. M. ChemSusChem 2016, 9, 644.

[46] Shaygan, N. A.; Rana, S.; Dohler, D.; Jirsa, F.; Meister, A.; 
Guadagno, L.; Koslowski, E.; Bron, M.; Binder, W, H. Chem.-Eur. J. 2015, 21, 10763.

[47] Li, Z.; Zhang, W.; Zhao, Q.; Gu, H.; Li, Y.; Zhang, G.; Zhang, F.; Fan, X. ACS Sustainable Chem. E

[48] Gaikwad, V. V.; Vitthal, B.; Saptal.; Harada, K.; Sasaki, T.; Daisuke, N. H.; Bhalchandra, M. B. ChemNanoMat 2015, 1, 489

[49] Xue, B.; Liang, X.-Y.; Liu, N.; Xu, T.-C.; Xu, J.; Li, Y.-X. Colloids Surf., A 2018, 538, 534.

[50] Sadjadi, S.; Heravi, M.; Raja, M. Int. J. Biol. Macromol. 2019, 122, 228.

[51] Zhu, J.; Wang, S.-Q.; Gu, Y.-K.; Xue, B.; Li, Y.-X. Mater. Chem. Phys. 2018, 208, 68

[52] Dai, W.-L.; Chen, L.; Yin, S.-F.; Luo, S.-L.; Au, C.-T. Catal. Lett. 2010, 135.

[53] Cheng, W.-G.; Chen, X.; Sun, J.; Wang, J.-Q.; Zhang, S.-J. Catal. Today 2013, 117.

[54] Adam, F.; Appaturi, J. N.; Ng, E. J. Mol. Catal. A, Chem. 2014, $386,42$.

[55] Yuan, C.; Huang, Z.; Chen, J. Catal. Commun. 2012, 24, 56.

[56] Hu, Y.; Tang, S.; Jiang, L.; Zou, B.; Yang, J.; Huang, H. Proc. Biochem. 2012, 47, 2291.

[57] Setyawan, H.; Balgis, R. Asia-Pac. J. Chem. Eng. 2012, 7, 448.

[58] Yang, J-B.; Zeng, T.; Cai, D-R.; Li, L.; Tang, W-L.; Hong, R-Y.; Qiu, T. Asia-Pac. J. Chem. Eng. 2016, 11, 901 .

[59] Guo, L.-Y.; Deng, L.-L.; Jin, X.-C.; Wu, H.; Yin, L-Z. Catal. Lett. 2017, 147, 2290 .

[60] Wang, Y.-Q.; Zhao, D.; Wang, L.-L.; Wang, X.-Q.; Li, L.-J.; Xing, Z.-P.; Ji, N.; Liu, S.-J.; Ding, H. Fuel 2018, 216, 364.

[61] Hierro, I.; Pérez, Y.; Fajardo, M. M. Mesoporous Mater. 2018, 263, 173

[62] Alcañiz, J J.; Gascon J.; Kapteijn, F. J. Mater. Chem. 2012, 22, 10102.

[63] Dhakshinamoorthy, A.; Opanasenko, M.; Čejka, J.; Garcia, H. Catal. Sci. Technol. 2013, 3, 2509.

[64] Tanabe, K. K.; Cohen, S. M. Inorg. Chem. 2010, 49, 6766.

[65] Valenzano, L.; Civalleri, B.; Chavan, S.; Palomino, G. T.; Areán, C. O.; Bordiga. S. J. Phys. Chem. C 2010, 114, 11185.

[66] Gao, W.-Y.; Chen, Y.; Niu, Y.; Williams, K.; Cash, L.; Perez P. J.; Wojtas, L.; Cai, J.; Chen, Y-S.; Ma, S. Angew. Chem. 2014, 126, 2653.

[67] Liang, J.; Xie, Y.-Q.; Wang, X.-S.; Wang, Q.; Liu, T.-T.; Huang, Y.-B.; Cao, R. Chem. Commun. 2018, 54, 342

[68] Shaabani, A.; Mohammadian, R.; Farhid, F.; Alavijeh, M. K.; Amini, M. M. Ind. Eng. Chem. Res. 2019, 58, 2784.

[69] Chong, S.-Y.; Wang, T.-T.; Cheng, L.-C.; Lv, H.-Y.; Ji, M. Lang- muir 2019, 35, 495.

[70] Liu, Y.-Z.; Ma, Y.-H.; Zhao, Y.-B.; Sun, X.-X.; Gándara, F.; Furukawa, H.; Liu, Z.; Zhu, H.-Y.; Zhu, C.-H.; Suenaga, K.; Oleynikov, P.; Alshammari ,A. S.; Zhang, X.; Terasaki, O.; Yaghi, O. M. Science 2016, 351, 365.

[71] Feng, X.; Ding, X.; Jiang, D. Chem. Soc. Rev. 2012, 41, 6010.

[72] Ding, S.-Y.; Wang, W. Chem. Soc. Rev. 2013, 42, 548.

[73] Dogru, M.; Bein, T. Chem. Commun. 2014, 50, 5531.

[74] Liu, X.-H.; Guan, C.-Z.; Wang, D.; Wan, L.-J. Adv. Mater. 2014, 26, 6912.

[75] Sun, L.; Boo, W. J.; Sue, H.-J.; Clearfield, A. New J. Chem. 2007, $31,39$.

[76] Sun, L.-Y.; O’Reilly, J. Y.; Kong, D.-Y.; Su, J.-Y.; Boo, W. J.; Sue, H-J.; Clearfield, A. J. Colloid Interface Sci. 2009, 333, 503.

[77] Tang, M.; Yang T.-S.; Zhang, Y. Sci. China, Technol. Sci. 2016, 59, 436.

[78] Wei, S.-Y.; Lizu, M.; Zhang, X.; Sampathi, J.; Sun, L.-Y.; Milner, M. F. High Perform. Polym. 2013, 25, 25.

[79] Zhou, Y.; Huang, R.; Ding, F.; Brittain, A. D.; Liu, J.; Zhang, M.; Xiao, M.; Meng, Y.; Sun, L. ACS Appl. Mater. Interfaces 2014, 6, 7417.

[80] Zhou, Y.; Wang, A.; Wang, Z.; Chen, M.; Wang, W.; Sun, L.; Liu, X. RSC Adv. 2015, 5, 93969.

[81] Zhou, Y.; Liu, J.; Xiao, M.; Meng, Y.; Sun, L. ACS Appl. Mater. Interfaces 2016, 8, 5547.

[82] He, X.; Xiao, H.; Choi, H.; Díaz, A.; Mosby, B.; Clearfield, A.; Liang, H. Colloids Surf. A 2014, 452, 32.

[83] Gower, L. B. Chem. Rev. 2008, 108, 4551.

[84] Dash, M.; Chiellini, F.; Ottenbrite, R. M.; Chiellini, E. Prog. Polym. Sci. 2011, 36, 981.

[85] Ifuku, S.; Miwa, T.; Morimoto, M.; Saimotoa, H. Green Chem. 2011, 13, 1499.

[86] Chung, K.-H.; Cho, M. Y.; Sung, M.-H.; Poo, H.; Lim, Y. T. Chem Commun. 2011, 47, 8889.

[87] Dong, B.; Wang, L-Y.; Zhao, S.; Ge, R-L.; Song, X.-D.; Wang, Y.; Gao, Y.-A. Chem. Commun. 2016, 52, 7082.

[88] Zhou, Y.-J.; Liu, J.-J.; Huang, R.-C.; Zhang, M.; Xiao, M.; Meng, Y.-Z.; Sun, L.-Y. Dalton Trans. 2017, 46, 13126.

[89] Sun, J.; Wang, J.-Q.; Cheng, W.-G.; Zhang, J.-X.; Li, X.-H.; Zhang, S.-J.; She, Y.-B. Green Chem. 2012, 14, 654.

[90] Nassor, E.; Mambrini, R. V.; Santos, E.; Moura, F.; Araujo, M. H. J. Inorg. Organomet. Polym. Mater. 2018, 28, 2288.

[91] Zhang, M.-J.; Tang, Z.-Y.; Fu, W.-Q.; Wang, W.-Y.; Tan, R.; Yin, D.-H. Chem. Commun. 2019, 55, 592. 